\title{
BMJ Open SToP (See, Treat, Prevent) skin sores and scabies trial: study protocol for a cluster randomised, stepped-wedge trial for skin disease control in remote Western Australia
}

\author{
Marianne J Mullane, ${ }^{1}$ Timothy C Barnett, ${ }^{1}$ Jeffrey W Cannon, ${ }^{1}$ \\ Jonathan R Carapetis, ${ }^{1,2,3}$ Ray Christophers, ${ }^{4}$ Juli Coffin, ${ }^{5,6}$ Mark A Jones, ${ }^{\oplus} 1$ \\ Julie A Marsh, ${ }^{1}$ Frieda Mc Loughlin, ${ }^{1}$ Vicki O'Donnell, ${ }^{7}$ Rebecca Pavlos, ${ }^{1}$ \\ Bec Smith, ${ }^{8}$ Andrew C Steer, ${ }^{9,10,11}$ Steven Y C Tong, ${ }^{12,13}$ Roz Walker, ${ }^{1}$ \\ Asha C Bowen ${ }^{\oplus 1,2,3,13}$
}

To cite: Mullane MJ, Barnett TC Cannon JW, et al. SToP (See, Treat, Prevent) skin sores and scabies trial: study protocol for a cluster randomised, stepped-wedge trial for skin disease control in remote Western Australia. BMJ Open 2019:9:e030635. doi:10.1136/ bmjopen-2019-030635

- Prepublication history and additional material for this paper are available online. To view these files, please visit the journal online (http://dx.doi. org/10.1136/bmjopen-2019030635).

Received 24 March 2019 Revised 29 August 2019 Accepted 30 August 2019

D Check for updates

(c) Author(s) (or their employer(s)) 2019. Re-use permitted under CC BY-NC. No commercial re-use. See rights and permissions. Published by BMJ.

For numbered affiliations see end of article.

Correspondence to Dr Asha C Bowen; asha.bowen@telethonkids. org.au

\section{ABSTRACT}

Introduction Skin is important in Australian Aboriginal culture informing kinship and identity. In many remote Aboriginal communities, scabies and impetigo are very common. Untreated skin infections are painful, itchy and frequently go untreated due to under-recognition and lack of awareness of their potential serious complications. We hypothesise that the skin infection burden in remote Aboriginal communities can be reduced by implementing streamlined training and treatment pathways integrated with environmental health and health promotion activities, tested in the See, Treat, Prevent (SToP skin sores and scabies) trial.

Methods and analysis SToP will evaluate a skin control programme using a stepped-wedge, cluster randomised trial design with three intervention components (the 'SToP activities'): (1) seeing skin infections (development of training resources implemented within a community dermatology model); (2) treating skin infections (employing the latest evidence for impetigo, and scabies treatment); and (3) preventing skin infections (embedded, culturally informed health promotion and environmental health activities). Four community clusters in the remote Kimberley region of Western Australia will participate. Following baseline data collection, two clusters will be randomly allocated to the SToP activities. At 12 months, the remaining two clusters will transition to the SToP activities. The primary outcome is the diagnosis of impetigo in children (5-9 years) at school-based surveillance. Secondary outcome measures include scabies diagnosis, other child health indicators, resistance to cotrimoxazole in circulating pathogenic bacteria, determining the economic burden of skin disease and evaluating the cost effectiveness of SToP activities. Ethics and dissemination This study protocol was approved by the health ethics review committees at the Child and Adolescent Health Service (Approval number RGS0000000584), the Western Australian Aboriginal Health Ethics Committee (Reference number: 819) and the University of Western Australia (Reference RA/4/20/4123).
Strengths and limitations of this study

- The cluster randomised trial with stepped-wedge design was chosen to engage as many people as possible in the activities of the trial, while providing the pathway for rapid translation of results into other communities across remote Australia if successful.

- Through the partnerships established with local clinical and environmental health service providers, this study is well positioned to see a substantial, sustained reduction in the burden of skin infections.

- This study is a multifaceted intervention in communities that face a range of adversities and changing contexts and, as such, it may be difficult to tease out the individual components leading to successful skin infection control.

Study findings will be shared with community members, academic and medical communities via publications and presentations, and in reports to funders. Authorship for all publications based on this study will be determined in line with the Uniform Requirements for Manuscripts Submitted to Biomedical Journals published by the International Committee of Medical Journal Editors. Sharing results with organisations and communities who contributed to the study is paramount. The results of the SToP trial will be shared with participants in a suitable format, such as a single summary page provided to participants or presentations to communities, the Kimberly Aboriginal Health Planning Forum Research Subcommittee and other stakeholders as appropriate and as requested. Communication and dissemination will require ongoing consultation with Aboriginal communities to determine appropriate formats. Trial registration number ACTRN12618000520235.

\section{INTRODUCTION}

Skin is important in Aboriginal culture as it is central to identity, determines kinship, 
connection to country and creates belonging. ${ }^{1}$ Maintaining healthy skin is important for maintaining overall physical and cultural health and well-being. However, environmental, social and demographic factors place Australian Aboriginal people living in remote communities at risk of skin infections including impetigo, ${ }^{2}$ scabies $^{3}$ and crusted scabies, ${ }^{45}$ and their sequelae. These skin infections have a high burden and contribute to sustaining the health disparities experienced by Australian Aboriginal people. ${ }^{67}$ Australian Aboriginal children living in remote communities have the highest burden of impetigo in the world, with a median prevalence of $45 \%$ (IQR 34\%-49\%), surpassing Africa (7\% (IQR 4\%-12\%)), Asia (7\% (IQR 3\%-16\%)) and Oceania $(30 \%($ IQR $15 \%-42 \%)) .{ }^{6}$ Scabies has a similarly high burden, with the prevalence reported at 5\%-35\% in remote Australian contexts. ${ }^{68}$ Australian Aboriginal children have the third highest global prevalence of infestation by scabies, behind only Panama and Fiji (at $78 \%$ and $44 \%$ children infested, respectively). ${ }^{8}$ Skin infections are not benign. Untreated skin infections may lead to group A streptococcal (GAS) ${ }^{9}{ }^{10}$ and staphylococcal sepsis, ${ }^{11} 12$ skeletal infection, ${ }^{13-16}$ and postinfectious complications of GAS, which include chronic kidney disease ${ }^{17} 18$ and potentially rheumatic heart disease (RHD) ${ }^{19}$ GAS-related kidney disease and RHD occur in Australian Aboriginal people at among the highest rates in the world. ${ }^{20}$ Skin infections are potentially the most remediable cause of serious disease in remote living Australian Aboriginal people.

In remote Western Australian communities, skin infections are the second most common cause for primary care presentations $^{21}$ and account for approximately $1 \%$ of all hospitalisations in childhood, with $15 \%$ of Aboriginal infants admitted to hospital with a skin infection before their first birthday. ${ }^{22}$ Aboriginal children are almost 15 times more likely to be hospitalised with skin infections than their non-Aboriginal peers and Aboriginal children living in the Kimberley region suffer from the highest hospitalisation rate for skin infections in Western Australia. $^{22}$

Despite the burden and serious consequences, skin infections have become 'normalised' and frequently go untreated due to under-recognition, lack of awareness of their importance and inadequate knowledge of evidencebased, effective treatment. ${ }^{23}$ Comprehensive and sustainable skin disease control in remote Aboriginal contexts is urgently needed. The aim of this study is to evaluate whether a combination of improved training, provision of evidencebased treatment in a treatment as prevention framework, environmental health and health promotion activities will result in a sustained reduction in skin infection in remote Western Australian Aboriginal communities.

\section{METHODS AND DESIGN}

\section{Study design}

The See, Treat, Prevent (SToP skin sores and scabies) trial is a pragmatic, cluster randomised trial involving four community clusters with an open-cohort stepped-wedge design. $^{24-26}$ The clusters receive the intervention at different timepoints. The order in which the clusters start the intervention is randomised and data are collected from the clusters over time. During a lead-in period (table 1, shaded white-visits 1 and 2), baseline skin infection data will be collected from school-based surveillance study visits. The school-based surveillance will be augmented with routinely available clinic data from electronic records, hospital records and baseline skin health Knowledge, Attitudes and Practice surveys collected as part of a process evaluation of the trial (see online supplementary file 1). At the start of visit 3, the SToP interventions ('SToP activities') will commence in two of the clusters. The remaining two clusters will transition to the interventions 12 months later (at visit 6). By the end of the trial, all four clusters will be receiving the SToP activities. The final school-based surveillance measurement will be completed at visit 9, and maintenance activities will commence (table 1-shaded yellow). Maintenance activities will be an enhanced business as usual approach, where we expect that the communities will continue to use the SToP trial resources and processes but without further education and input from the trial team. During the 12-month maintenance period (table 1-year 4), routinely available clinic/hospital data will continue to be collected to investigate the longer term impact of the intervention in the absence of regular school-based surveillance and to evaluate the intervention effectiveness on indicators of chronic disease. No data will be collected at timepoints 4 nor 8 because the communities are inaccessible at that time of year (table 1 ).

SToP activities comprise three components:

1. Seeing skin infections through the development of training resources within a community dermatology model $^{27}$ through school-based surveillance of the primary outcome measure.

2. Treating skin infections using the most recent, evidence-based approach: cotrimoxazole two times a day for 3 days for impetigo, ${ }^{28}$ ivermectin on days 0 and 8 for scabies cases and day 0 for their contacts ${ }^{29}$ and holistic care including treatment of those identified with crusted scabies (ivermectin on days 0,1 and 8 for grade 1 crusted scabies $)^{430}$.

3. Preventing skin infections through developing and embedding, culturally informed health promotion and environmental health activities. ${ }^{31}$

\section{Study setting}

The four clusters in this trial are single remote Australian Aboriginal communities, or groups of communities in close proximity, within distinct geographical areas in the Kimberley region of far north Western Australia. The populations range from approximately 450 people in the smallest discrete community to approximately 1000 in the largest clusters. The distance from the communities to the nearest major regional town ranges from 200 to $1200 \mathrm{~km}$. The Kimberley region is one of the most remote and sparsely populated in Australia, with an estimated 


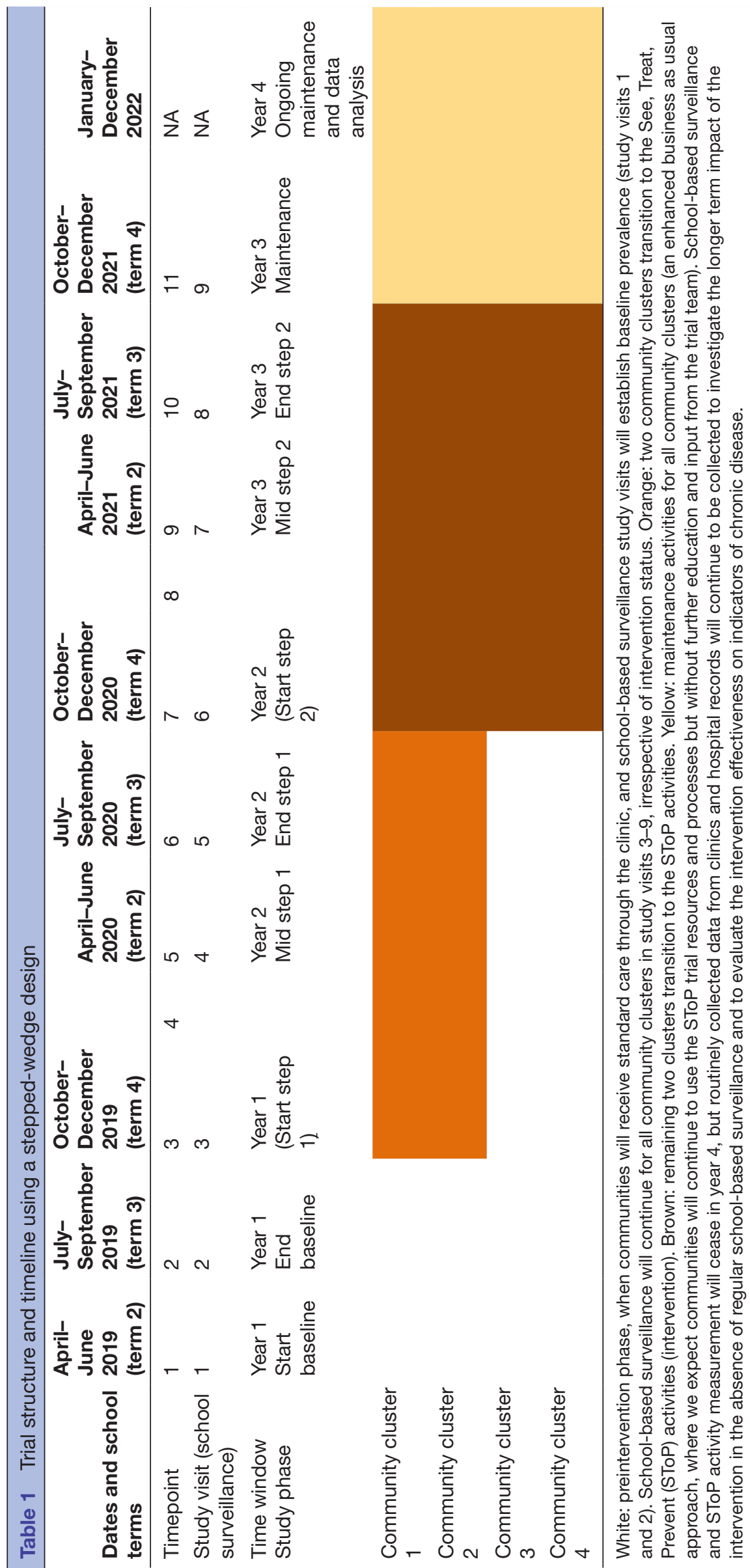


population of approximately 35000 people, $47 \%$ of whom are Aboriginal, spread over $421000 \mathrm{~km}^{2} .{ }^{27}$ The Kimberley Aboriginal Medical Service and Western Australian Country Health Services (Kimberley) administer the community clinics, staffed by remote area nurses, general practitioner and Aboriginal health practitioners. The planned trial period is between April 2019 and December 2021 with the intervention planned for delivery between September 2019 and September 2021. Maintenance will commence in October 2021 (table 1) and continue to December 2022.

\section{Participants/eligibility criteria}

Trial participants will be recruited from four community clusters. All members of the participating communities may be exposed to the SToP activities. Community clusters were chosen based on the following pragmatic criteria: a population of approximately 1000 people, access to a health clinic staffed full time by nurses, practical accessibility for research staff and with advice from local Aboriginal and non-Aboriginal health and environmental health service leaders from the Kimberley Aboriginal Health Planning Forum. The Kimberley Aboriginal Health Planning Forum is a state government endorsed committee that meets monthly to discuss strategic priorities for delivery of healthcare for Aboriginal people throughout the Kimberley region. With advice from the Chief Investigator on the criteria for community clusters, the leaders of the Kimberley Aboriginal Health Planning Forum recommended the most appropriate communities for inclusion, prior to contact with community elders. During the 12-month period of community engagement that preceded ethics submissions, consultation with leaders of the Kimberley Aboriginal Health Planning Forum and community elders in the nine communities confirmed the appropriateness of these selections. Communities were excluded if community leaders declined to participate.

\section{Definitions}

Impetigo (commonly referred to as 'skin sores' or 'school sores') is the result of a bacterial infection usually caused by Staphylococcus aureus and/or GAS. ${ }^{32}$ Clinically, impetigo typically presents as three subclassifications that generally follow the progression from initial infection as the sore heals-(1) 'purulent' where the sores start as round/oval pus-filled bumps that may progress into blisters (figure 1); (2) 'crusted' where the sore produces a clear honey-coloured fluid that forms a crust on the skin (figure 2); (3) 'flat/dry' when the crust resolves and the healing skin beneath the preceding crust may initially be pink and flat as the sore heals (figure 3). Early on, the flat dry sores may have resolving peeling of the edges. Later, this becomes a rounded, flat scar-evidence of preceding impetigo. Scabies is an infection of the skin as a result of an infestation by the mite Sarcoptes scabiei var. hominis. ${ }^{32}$ It results in small papules $(1-2 \mathrm{~mm})$ that are intensely itchy. Scratching

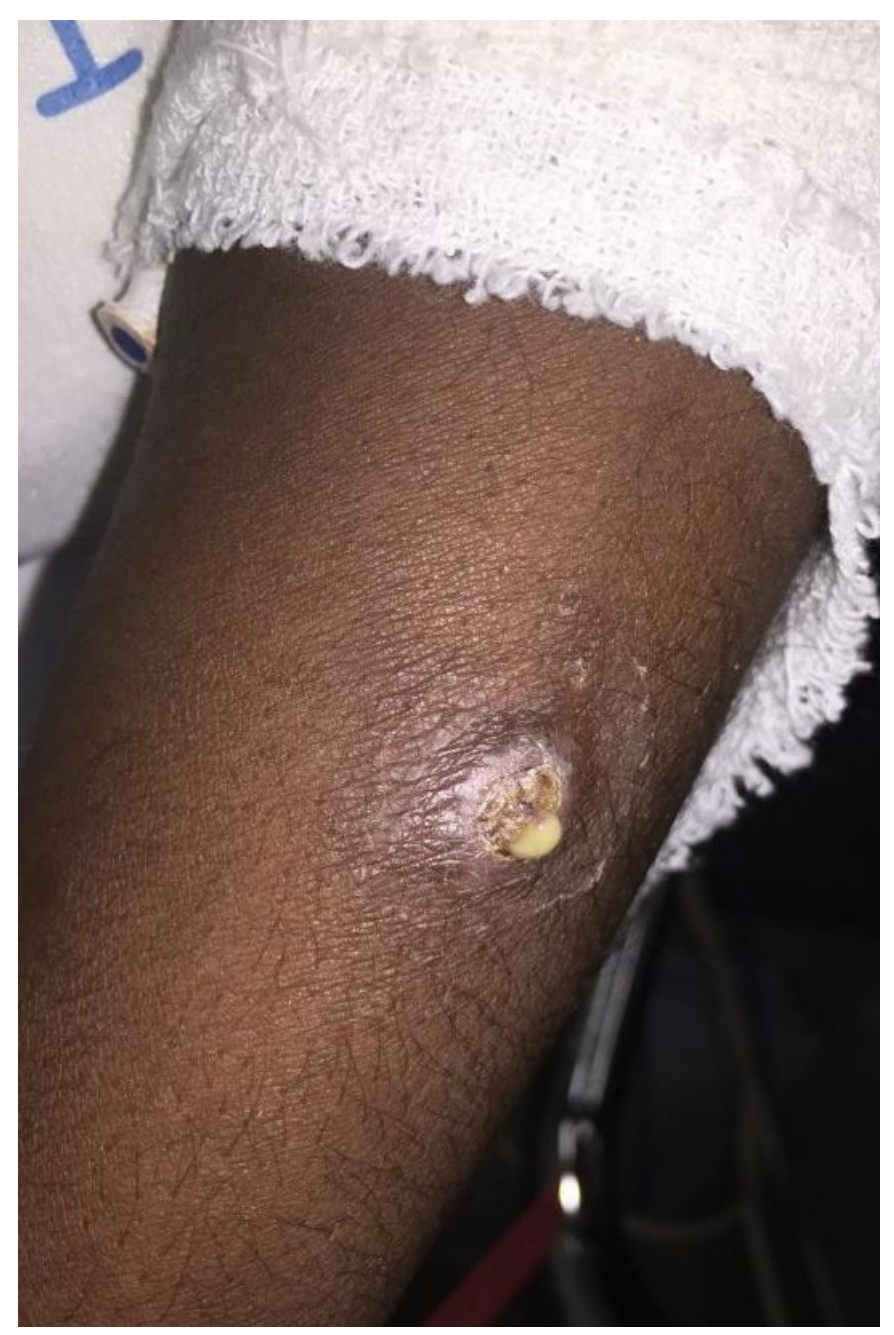

Figure 1 Photograph of a purulent skin sore. Classical features include the presence of yellow, turbid fluid dripping from the lesion (pus), and a round crusted scab approximately $1 \mathrm{~cm}$ in diameter. Source: National Healthy Skin Guideline for the Prevention, Treatment and Public Health Control of Impetigo, Scabies, Crusted Scabies and Tinea for Indigenous Populations and Communities in Australia (1st edition), 2018 (publicly available).

due to scabies can break the skin barrier resulting in secondary bacterial infection. Crusted scabies is a severe form of scabies (hyperinfestation) where the person's immune system cannot control the infestation and the mites rapidly multiply. The skin reacts to the infestation by crusting and shedding.

\section{Screening and registration}

In preparation for the commencement of the SToP trial, extensive community consultation has been underway in the Kimberley since January 2017, in partnership with the Kimberley Aboriginal Medical Service and Western Australian Country Health Services (Kimberley).

Following community consultation activities and recruitment of communities, formal consent to participate in the trial was provided by the authorised community representatives in a letter of support. 


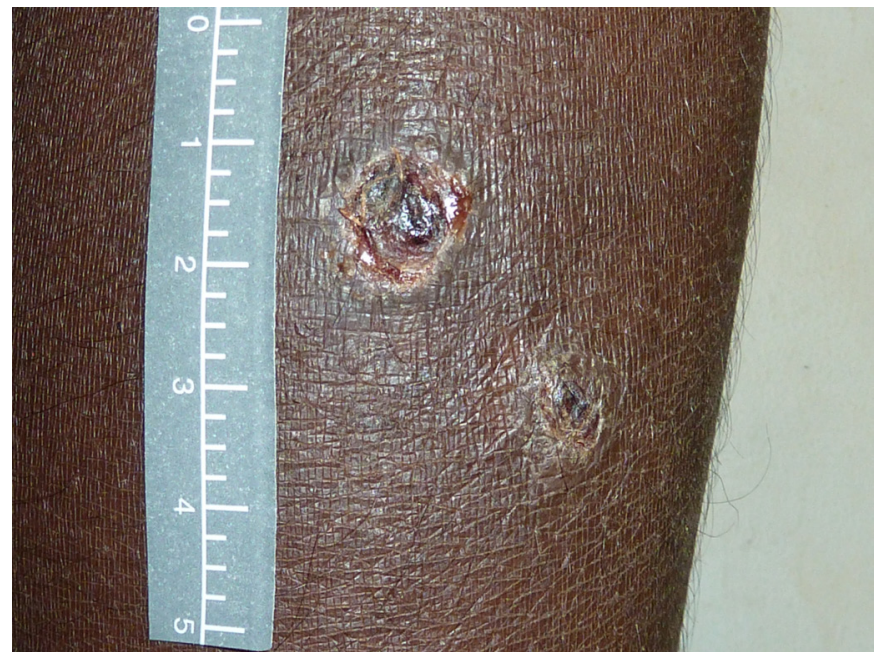

Figure 2 Photograph of a crusted skin sore. Classical features include a $1 \mathrm{~cm}$, rounded lesion with thick crust present. Early peeling of the sore edges can be appreciated. There is a second rounded, crusted lesion distal to this also seen. Source: National Healthy Skin Guideline for the Prevention, Treatment and Public Health Control of Impetigo, Scabies, Crusted Scabies and Tinea for Indigenous Populations and Communities in Australia (1st edition), 2018 (publicly available).

\section{Randomisation and allocation concealment}

The community cluster, rather than individuals, is the unit of randomisation. Community clusters will be randomly allocated to either the first wave (two clusters in step 1) or second wave (remaining two clusters in step 2) by the trial

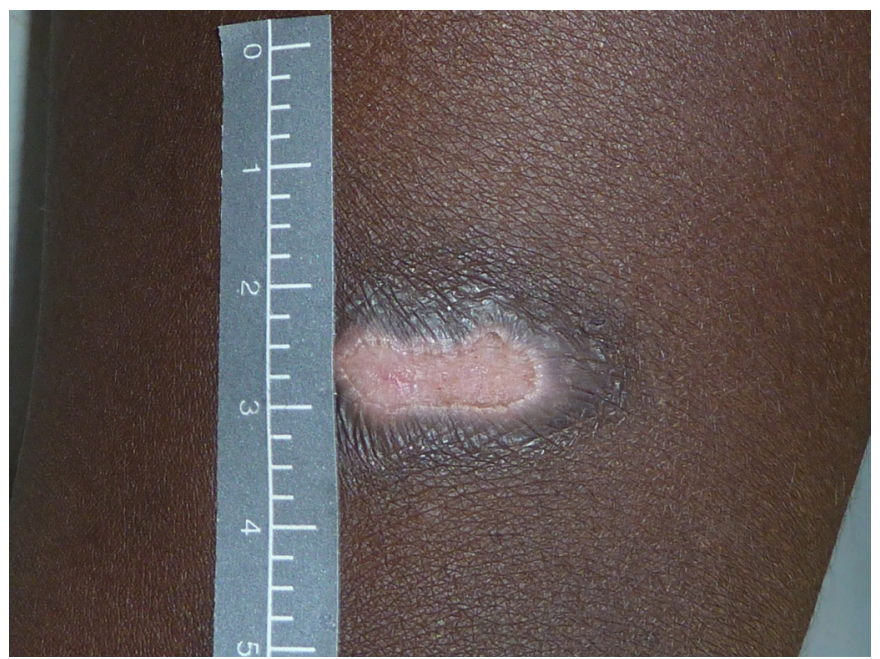

Figure 3 Photograph of a recently healed skin sore also known as a flat, dry sore. The lesion is pale pink, has evidence of tethering at the edges and the crust has completely resolved. This lesion will continue to improve with the pale pink new skin eventually completely epitheliarising with a thin, flat, round lesion present as evidence of the preceding skin sore. Source: National Healthy Skin Guideline for the Prevention, Treatment and Public Health Control of Impetigo, Scabies, Crusted Scabies and Tinea for Indigenous Populations and Communities in Australia (1st edition), 2018 (publicly available). statistician using a computer program with a validated random number generator.

\section{Blinding}

This is an open-label study.

\section{Intervention group}

The intervention groups are the community clusters that have been randomised to receive the SToP activities. In step 1 (visits 3-5; table 1), two randomly selected community clusters will receive the SToP activities. In step 2 (visits 6-8; table 1), the remaining two community clusters will transition to receive the SToP activities.

\section{Control group}

Each community cluster acts as its own control (preintervention and postintervention) throughout the course of the study. The community's standard care of skin infections (table 2) will continue while it remains in the preintervention phase. $^{33}$ SToP trial-led training, treatment, environmental health and health promotion activities will not occur during this period.

\section{Intervention components (SToP activities) \\ 'See' surveillance}

School-based surveillance activities hold a dual purpose for this study: measurement of the primary outcome, and completion of skin identification training activities as per the community dermatology model (see the Training section). School-aged children have a high burden of disease and are an established cohort for surveillance purposes. School-based surveillance for all children (3-15 years old), and early childhood centres where feasible (for children $<5$ years of age), will occur in all four community clusters to establish baseline prevalence data for impetigo and scabies (study visits 1-2, table 1). School (and early childhood centres) surveillance will continue in all four community clusters during study visits 3-9 (table 1), irrespective of intervention or control status of the community. During surveillance, impetigo, scabies and crusted scabies will be assessed via a visual diagnosis for each participant; impetigo meeting the definitions of purulent, crusted or flat/dry features, scabies being the presence of papules, burrows and itch, and infected scabies defined as itch, papules/burrows with secondary evidence of crust or purulence. Suspected crusted scabies will be assessed in conjunction with local clinic health professionals as recommended by current local clinical guidelines. Surveillance data will be augmented with data from clinic presentations for skin infections to develop dashboard reporting of skin infections for each community cluster. Deidentified clinic level data on the number of school referrals received and type of treatment administered/dispensed will also be collected from the electronic clinical record.

\section{'See' training}

Training will occur prior to each surveillance visit using a custom-made SToP trial version of the National Healthy 
Table 2 First-line therapy recommended in the Kimberley standard treatment guidelines ${ }^{33}$ compared with the See, Treat, Prevent (SToP) trial treatment guidelines*

\begin{tabular}{lll}
\hline Condition & Kimberley Standard Treatment Guidelines & \\
\hline Impetigo & Benzathine penicillin G, intramuscular & SToP Trial Treatment Guidelines \\
\hline Scabies & Permethrin, topical & Cotrimoxazole, oral $^{28}$ \\
Crusted scabies & & Ivermectin, oral $^{29}$ \\
\hline
\end{tabular}

*See also table 3.

Skin Guideline resources. ${ }^{32}$ Local health workers will be paired with experienced clinicians and/or appropriately trained study staff to examine all eligible children and document the prevalence of impetigo, which is the primary outcome assessment. Scabies prevalence will be documented as a secondary outcome. The diagnosis and holistic management of crusted scabies will occur using a trial-specific version of the protocol developed by One Disease ${ }^{30}$ which incorporates crusted scabies within the chronic disease model of care for all patients presenting to clinic. The regular repeated school screening throughout the trial (table 1) will provide refresher training and targeted upskilling of new health, school and community workers. Additionally, online training manuals and videos have been developed that incorporate self-assessment materials based on a collection of photographs of skin infections for current and future health workers beyond the trial. ${ }^{34}$

\section{'Treat'}

Children identified during surveillance as requiring treatment for skin infection will be referred to the clinic for treatment (table 2). Treatment will be as per the standard Kimberley treatment guidelines for skin infection ${ }^{33}$ until the SToP activities start in step 1. Alternative treatments will be administered if the study treatments are contraindicated (table 3 ).

This trial will use ivermectin off label as the first-line treatment for cases of scabies and where possible, singledose preventative administration of ivermectin for asymptomatic, household contacts of scabies cases. Crusted scabies will be treated according to the label for use in Australia (Ivermectin Public Summary Document, March 2014).$^{35}$ The use of ivermectin as the first-line treatment of scabies is not currently routine practice in remote clinics in Western Australia, but recent high-quality clinical trials have demonstrated superior efficacy of ivermectin for treatment and prevention of scabies. ${ }^{29}$ Therefore, ivermectin will be implemented via Structured Administration and Supply Arrangements under the Western Australian Public Health Act 2016 to streamline access to the best evidence-based treatment available. ${ }^{36}$

'Prevent' - health promotion and environmental health activities Health promotion and environmental health activities will supplement the well-established activities led by the research partner organisations, Kimberley Aboriginal Medical Services, Western Australia Country Health

Table 3 Regimens for skin infection treatment in the See, Treat, Prevent trial*

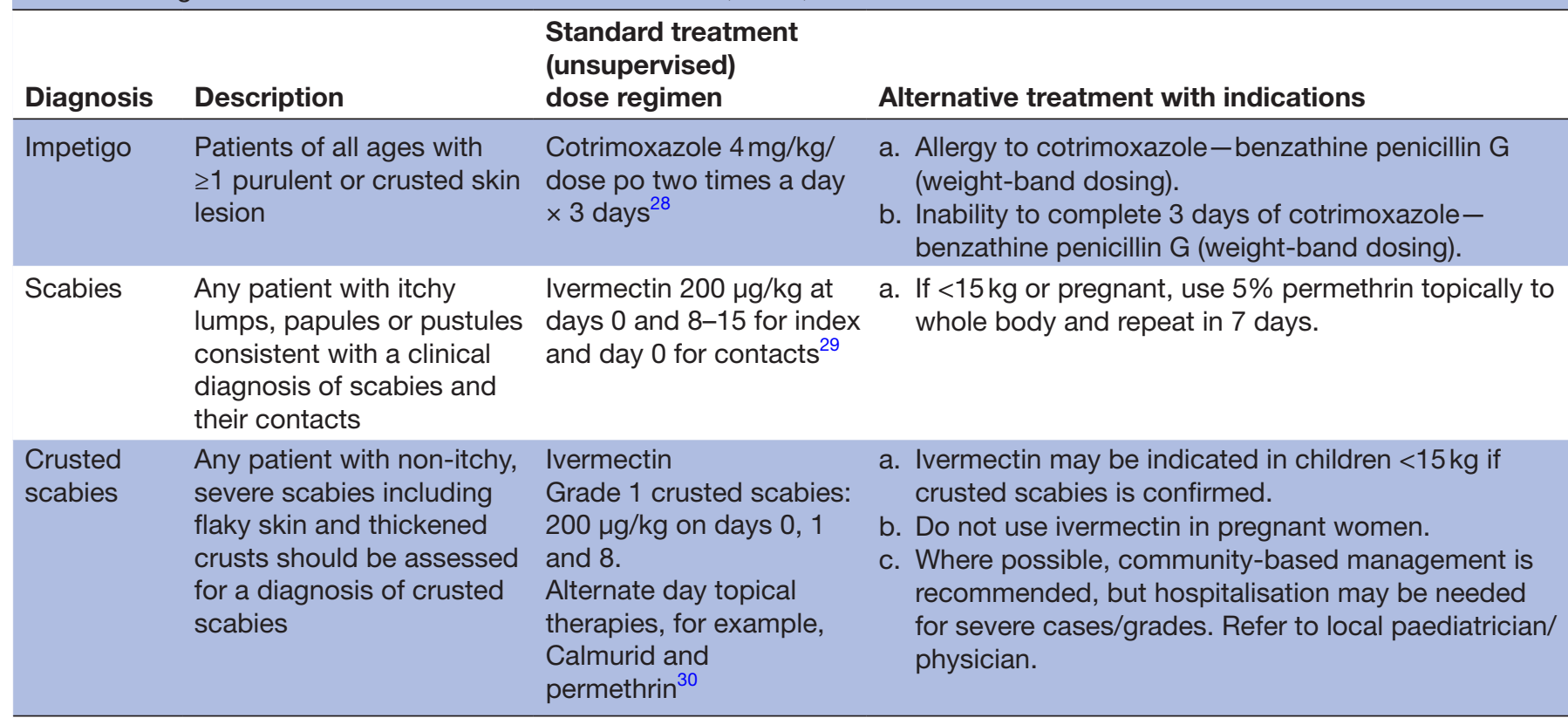

*Alternative options for those unable to comply with the standardised, evidence-based treatment. 
Services (Kimberley) and the Nirrumbuk Environmental Health and Services. All prevention research activities will be integrated with usual service delivery and enhanced where possible to improve the delivery of health promotion and environmental health activities at the time of the study surveillance visits.

Commencing during the consultation phase and continuing through the trial, community participatory action research ${ }^{37}$ will be used to engage community members and service providers in defining the format (including online, social media, posters, flipcharts, mobile phone messaging, music videos, public service announcements) and content of health promotion materials. The messages will focus on the importance of seeking healthcare for skin infections as well as appropriate prevention strategies (including good skin hygiene, insect bite prevention, care of minor trauma and maintenance of skin integrity). ${ }^{32}$ In addition, a music video that aims to improve community awareness of the importance of environmental health on skin and overall health will be produced during step 1 for ongoing health promotion.

Other environmental health activities aim to strengthen the relationships between clinic and environmental health services. Wherever possible, environmental health service staff will complete training and surveillance activities in partnership with the study team. Children identified with skin infections during surveillance will receive two referrals: one to the clinic for treatment and one to environmental health services for a home assessment.

Timing and duration of the SToP interventions are provided in the online supplementary file 2.

\section{Patient and public involvement}

Community and local stakeholder involvement were actively sought during the design and preparation phase of this trial and will continue to be critical in informing the trial as it progresses. Community representatives and/ or reference groups will be formed in consultation with communities and guide the implementation and analysis of the trial.

\section{Objectives}

Primary objective (table 4).

The primary objective is to achieve a $50 \%$ reduction in the prevalence of impetigo in school-aged children (5-9 years) from baseline (visit 1,2019 ) to maintenance (visit 9, 2021)

Secondary objectives are outlined in table 4 . We will use routinely collected data from clinics and hospital records in the maintenance phase (table 1) to investigate the longer term impact of the intervention in the absence of regular school-based surveillance and to evaluate the intervention effectiveness on indicators of chronic disease.

\section{Evaluation}

In addition to measurement of the above outcomes, a process evaluation will explore the way in which the SToP activities are implemented. This will evaluate whether implementation was delivered as intended and provide insights into the practice changes necessary to translate this trial more broadly (see online supplementary file 1). A prespecified evaluation and monitoring framework was developed for the SToP trial using programme theory $^{38}{ }^{39}$ (see online supplementary file 1 ). These measures will determine whether any outcomes arise as a result of the SToP activities and what intervention elements contributed to its success or failure. The monitoring and evaluation framework developed for the trial will collect evaluative data using mixed methods (qualitative and quantitative) concurrently with the trial outcome measures, and where possible, use existing sources of data and/or be paired with other study data collection procedures.

Quantitative evaluation data sources include case report forms from school surveillance, regional level data from the electronic health records, cluster-level environmental health service records, trial records and laboratory testing of skin swabs. Qualitative data will be derived from project officer reports, semistructured interviews, staff reflection logs, and Knowledge, Attitudes and Practice surveys. For qualitative analysis, codes will be developed de novo from the data without prespecified questions or assumptions. QSR International's NVivo 10 software will be used to analyse qualitative data. ${ }^{40}$

\section{Sample size calculation}

This cluster randomised trial with stepped-wedge design has two steps, two measurements during baseline, three in each step and a final visit to assess maintenance. Assuming baseline prevalence of impetigo to be $40 \%$ or higher implies that we will have $90 \%$ power to detect a $50 \%$ reduction in impetigo prevalence in school children aged 5-9 years in four large community clusters. ${ }^{41}{ }^{42}$ The calculations assume: (1) two clusters switch to the SToP activities at each step; (2) an average cluster size of 84 children aged 5-9 years in each community (based on communities of at least 1000 people, with approximately $12 \%$ aged 5-9 years and a daily school attendance rate of around $70 \%$ ); (3) a significance level of $5 \%$; (4) an intracluster correlation coefficient of 0.05 (this assumed value maximises the design effect and thus the sample size); and (5) absence of cluster by time interactions and similar time trends across all clusters.

\section{Statistical analysis}

The modelling approach extends the approach put forward by Hussey and Hughes ${ }^{26}$ and is aligned with the commentary from Hemming et al and Barker et al. ${ }^{25} 43$ Generalised linear mixed-effect regression modelling with small-sample correction ${ }^{44}$ will be used to estimate the ORs of impetigo diagnosis at school surveillance in the intervention period compared with the control period. These methods use both the within-cluster and between-cluster information to estimate the treatment effect. The reduction in prevalence will be estimated as a weighted average 
Table 4 Outcome measures

Primary outcome measures

1. Diagnosis of impetigo in children aged 5-9 years.

Case report forms (CRFs) from school-based surveillance data.

\section{Secondary outcome measures}

\section{Outcome measures for other child health indicators of skin infection burden:}

a. Diagnosis of scabies in children aged 5-9 years.

b. Diagnosis of impetigo at early childhood centres and the clinic in children aged 0-4 years.

c. Diagnosis of scabies at early childhood centres and the clinic in children aged 0-4 years.

d. Overall clinic presentations due to skin conditions including abscess in all age deciles.

e. Age at first scabies and impetigo diagnosis in the 12-month birth cohort.

f. All-cause clinic presentations and hospitalisations from the communities, including those for non-skin (eg, anaemia), skin related (eg, sepsis) and skin infections in children aged $<10$ years.
- CRFs from school-based surveillance data. Automated electronic medical record clinic data extractions for skin conditions for local clinics.

- 12-month birth cohort data from automated electronic medical record clinic data extractions for local clinics and hospitals.

- Number of children referred to clinic from SToP school surveillance and the number of referred children that present to clinic.

\section{Outcome measures for monitoring antimicrobial resistance of bacterial skin pathogens:}

a. Monitoring of antimicrobial resistance, including cotrimoxazole, penicillin and methicillin in circulating Staphylococcus aureus and GAS strains.
- Microbiological data and antibiotic resistance testing for trimethoprim and cotrimoxazole for all skin and throat swabs from surveillance.

- Routinely collected microbiological data for the emergence of antibiotic resistant strains in other priority microorganisms in the region.

b. Monitoring of antimicrobial prescribing for skin infections and other conditions.

- Prescribing data for BPG, cotrimoxazole and ivermectin from automated electronic medical record clinic data extractions and prescribing data from Kimberley Pharmacy Services.

c. Monitoring for changes in circulating S. aureus and GAS genotypes.
- Whole genome sequencing for a representative isolates derived from $S$. aureus and group A strep positive skin and throat swabs in 2(a) at baseline and the end of step 2 .

\section{Outcome measures for the economic burden and evaluation}

a. Determine the costs of impetigo and scabies to the health sector and the participant.
Resources used or lost to diagnose and/or manage impetigo and/ or scabies, collected from multiple sources, including study CRFs, electronic health/clinic records and trial evaluation interview data. Considerations include:

- Costs of management/treatment, for example, hospitalisations and length of stay, emergency department visits, primary care presentations, additional Investigations/tests, comorbidities.

- Productivity losses, the cost of absenteeism from normal activities or school, caregiver work due to impetigo and scabies.

- Number and cost of environmental health referrals for household assessments and repairs.

b. Evaluate the cost effectiveness and cost utility of the SToP Considerations and sources include: trial activities in reducing the prevalence of impetigo and scabies skin infections and related complications. These outcomes will be used in both the within trial and modelbased cost effectiveness and cost utility analyses.
- Cost of resources used to deliver the SToP intervention activities (sources include trial records and budget).

- For within-trial cost-effectiveness analyses, calculation of cost per case of impetigo, of scabies infection, of other bacterial skin infections and of skin-related bacterial infections (collectively) averted.

- For within-trial cost-utility analyses, calculation of cost per disability-adjusted life year averted.

- Literature review for model-based analyses.

BPG, Benzathine penicillin G; GAS, group A streptococcal; SToP, See, Treat, Prevent.

of the treatment effect calculated across all communities. Community-level effects will also be reported. The model will include the presence/absence of impetigo as the dependent variable, fixed effects for time since start of study, transition to intervention (indicator variable) and, if appropriate, interactions between community and time, and between community and intervention. The analysis will include random effects for clusters and repeat measures of individuals. Using this model, it will be possible to investigate whether the intervention effect 
and secular trends are common across communities. In the event of convergence issues, we will adopt analogous methods such as generalised estimating equations (GEE) that have fewer assumptions compared with GLMMs or Bayesian hierarchical models with sensible priors on variance components. ${ }^{45}$ However, GEEs provide only population-level inference and, as per a GLMM, will require small-sample correction.

Descriptive statistics will be used to summarise the secondary outcomes and the economic evaluation will be conducted from the health system perspective. Cost-effective analysis of adopting the SToP trial activities in an ongoing programme will be compared with the status quo. The inclusion of activities carried out by environmental health teams will depend on their uptake and ability of the statistical analysis to separate their effects from the other SToP activities. Deterministic and probabilistic sensitivity analyses of all variables will be used in the cost-effective analysis.

\section{Data and safety monitoring}

Data management and trial monitoring will be performed according to good clinical practice protocols, standard operating procedures and monitoring processes. A data safety and monitoring board has been established to oversee safety monitoring.

\section{Data collection}

Since internet accessibility is often unreliable in the trial communities, data from school surveillance and from the evaluation activities will be collected on deidentified paper case report forms and questionnaire forms. Data will subsequently be entered onto an electronic database (Medrio $)^{46}$ that has been developed for this study. Participant contact details collected from the case report forms will be stored in a secure, password-protected database (REDCap) ${ }^{47}$ and only accessible by authorised study staff. All documents will be stored securely and only accessible by trial staff and authorised personnel.

Children with impetigo will have bacterial skin swabs collected and processed for skin pathogens and antimicrobial susceptibility testing as per previously published methods. ${ }^{48} 49$ Throat swabs will be collected at baseline and maintenance for detection of $S$. aureus and GAS.

\section{DISCUSSION}

Healthy skin is a shared priority between Aboriginal community members and researchers in this research trial. The SToP trial has been designed to facilitate better skin and overall health for children and their families. There are many obstacles to achieving this goal, including underdiagnosis and underdetection (normalisation), ${ }^{23}$ population mobility, ${ }^{50}$ high burden, ${ }^{6}$ effective but poorly tolerated treatment, ${ }^{2851}$ lack of sustained community engagement, ${ }^{52}$ high workforce turnover, ${ }^{53}$ high acute healthcare needs and ongoing social determinants of health. The combination of these factors and the legacy of colonisation have left the Australian Aboriginal community with intergenerational trauma, ${ }^{545}$ a high burden of chronic disease and a myriad of competing priorities. ${ }^{31}$ Despite these challenges, the SToP trial is taking a comprehensive and strengths-based approach ${ }^{56}$ to overcome the combination of these many obstacles through health promotion, improved access to the best available treatments and engaging health professionals in a better understanding of the importance of healthy skin.

Skin infections in remote Aboriginal communities have been well described and have remained relatively static over more than two decades. ${ }^{6}$ Several skin infection control programme have been trialled in remote communities, both in Australia ${ }^{35} 5157$ and internationally. ${ }^{29} 58$ Regrettably, the initial benefits of these interventions in remote communities have not been sustained over the longer term, likely due to the need for an external workforce driving the change. ${ }^{51}$

The SToP trial design extends previous research programme $^{51}$ that demonstrated the importance of sustainability, respect, beneficence and partnership as key priorities. In this large, comprehensive trial, considerable investment has been made, consistent with Australian government guidelines concerning the ethical conduct of Indigenous research ${ }^{59}$ in developing the partnerships necessary to ensure the study resources and outcomes can be translated beyond the life of the trial. Using the latest evidence-based treatments in a 'treatment as prevention' framework, the SToP trial will be supported by the development of enhanced training materials and improved knowledge of activities to maintain healthy skin. The community engagement and consultation process of the SToP trial began more than 2 years prior to the start of the trial in an iterative way to inform cluster selection and study design, particularly the prevention activities. Building trust between the research team and the communities involved is critical to success of studies like this, particularly through partnering with primary healthcare providers and remote Aboriginal communities.

The cluster randomised trial with stepped-wedge design was chosen to limit research burden and improve research feasibility, while allowing for rapid translation of findings into clinical care. Due to the high risk that untreated skin infection poses for this population, this trial design provides the most ethical approach, with no participating community cluster missing out on the potential benefits of the SToP activities, while also allowing robust statistical methods (randomisation to intervention timing and hierarchical modelling) to be applied and maximising efficiency (project staff can provide support for each community cluster as they transition to the intervention phase). We have designed this trial with sustainability and transferability in mind so that the SToP activities can be delivered within existing healthcare structures that will require few additional resources. If successful, results of this trial could be rapidly translated to other communities throughout the region, as well as remote Aboriginal communities in other parts of Australia. 
The evidence base to inform the treatment as prevention strategies has been developed over the last decade by several of the lead authors. Specifically, cotrimoxazole has been shown to be non-inferior to intramuscular benzathine benzyl penicillin for impetigo with fewer side effects ${ }^{28}$; ivermectin mass drug administration was more effective than either permethrin mass administration or standard of care, ${ }^{29}$ and the work of the non-profit organisation One Disease has advanced our understanding of crusted scabies. ${ }^{30}$ We recently released the first edition of the National Healthy Skin Guideline for Aboriginal Australians $^{32}$ in May 2018. The guideline will aid in diagnosis and streamline treatment decisions for patients throughout in Australia. The results of this trial will inform subsequent editions of the National Healthy Skin Guideline, environmental health activities and their impact on human health and other primordial prevention strategies where current evidence is limited.

The limitations of the SToP trial include the inability to assign a specific intervention to a specific outcome due to the complex, multimodal activities rolled out concurrently throughout the course of the study. The SToP trial may also be limited by small number of clusters and fewer visits than planned due to community inaccessibility during the long, northern Australian wet season (November-April) when several communities will not be accessible by road. There is potential for cross over with implementation of study interventions, and within clusters prior to commencement. Population mobility is high in this region, such that infection transmission may be increased along with permeability of the interventions between clusters. We are unable to measure population mobility formally and as such this is a limitation that may impact on trial outcomes. Finally, the high turnover of clinical staff reported in remote communities ${ }^{53}$ will make the ongoing education plans challenging for the study to sustain.

\section{CONCLUSIONS}

This protocol describes the comprehensive approach to sustainable skin disease control in remote Australian Aboriginal communities. Results are expected in 2022.

\section{Participant consent}

Following community consultation activities and recruitment, formal letters of support have been provided from community councils and/or other appropriate community and organisation leaders/traditional owners who give permission on behalf of their community to participate in the trial. Community-level consent is appropriate for these components of the trial given they involve clinics, schools and community members.

Individual written parent/carer consent will be obtained for children to participate in the school-based skin surveillance activities by employed Aboriginal staff during the trial, and for the use of ivermectin for scabies treatment (see online supplementary files 3 and
4). Surveillance data will be augmented with data from clinic presentations for skin infections to develop dashboard reporting of skin infections for each community cluster. Individual consent is not required for these data as deidentified clinic/community-level data are the unit of analysis. Approvals from appropriate data custodians have been obtained to access these data.

\section{Author affiliations}

${ }^{1}$ Wesfarmers Centre for Vaccines and Infectious Diseases, Telethon Kids Institute, University of Western Australia, Nedlands, Western Australia, Australia

${ }^{2}$ Faculty of Health and Medical Sciences, University of Western Australia, Crawley, Western Australia, Australia

${ }^{3}$ Department of Paediatric Infectious Diseases, Perth Children's Hospital, Nedlands, Western Australia, Australia

${ }^{4}$ Nirrumbuk Environmental Health \& Services, Nirrumbuk Aboriginal Corporation, Broome, Western Australia, Australia

${ }^{5}$ Aboriginal Health, Telethon Kids Kimberley, Telethon Kids Institute, University of Western Australia, Broome, Western Australia, Australia

${ }^{6}$ Nulungu Research Institute, University of Notre Dame, Broome, Western Australia, Australia

${ }^{7}$ Kimberley Aboriginal Medical Services, Broome, Western Australia, Australia

${ }^{8}$ Western Australian Country Health Services-Kimberley, Broome, Western

Australia, Australia

${ }^{9}$ Tropical Diseases Research Group, Murdoch Childrens Research Institute, Parkville, Victoria, Australia

${ }^{10}$ Department of Paediatrics, University of Melbourne, Melbourne, Victoria, Australia

${ }^{11}$ Department of General Paediatrics, Royal Children's Hospital, Melbourne, Victoria, Australia

${ }^{12}$ Peter Doherty Institute for Infection and Immunity, Royal Melbourne Hospital and The University of Melbourne, Melbourne, Victoria, Australia

${ }^{13}$ Menzies School of Health Research, Casuarina, Northern Territory, Australia

Twitter Marianne J Mullane @M_Mullane, Timothy C Barnett @tim_barnett1, Jonathan R Carapetis @jcarapetis, Juli Coffin @julicoff, Rebecca Pavlos @ RebeccaPavlos, Steven Y C Tong @syctong, Roz Walker @RozWalker1 and Asha C Bowen@AshaBowen

Acknowledgements The authors are grateful to the communities who will participate in this study and their contributions to the design and implementation of this study. They acknowledge the support of their research partners: the Kimberley Aboriginal Medical Service, the Western Australia Country Health ServicesKimberley and Nirrumbuk Environmental Health \& Services. They would also like to thank those involved in implementing the SToP trial. Kristen White, Glenn Pearson, John Jacky, Janine McNamara, David Atkinson, Tom Snelling, Clancy Read, David Hendrickx, Rachael Donovan, Rhona Dawson, Rahaney Poelina, Tehani Mahony, Tracy McRae, Mark Gunnerson, Anna Schauer, Lucy Davidson and Katherine Middleton have all contributed to the development of this study. They thank Alison Carleton for drafting the protocol manuscript for submission.

Contributors Study concept and design were conducted by ACB, JRC and JAM. Critical revision of concept and design and intellectual input in the study protocol was done by all authors. Drafting of the manuscript was done by AB and MJM. Critical revision of the manuscript was performed by all authors. Study supervision is conducted by the partnership steering group: ACB, JRC, BS, VO and RC.

Funding This study is funded by the Western Australia Department of Health Future Health WA Third Year Initiative: Kimberley Healthy Skin Program (FHWAYR32015/16-KHS) and the National Health and Medical Research Council (NHMRC) Project Funding (GNT1128950). AB and SYCT are supported by NHMRC fellowships (1088735 and 1145033). TCB is supported by a Career Development Fellowship from the NHMRC-funded 'Improving Health Outcomes in the Tropical North: A multidisciplinary collaboration' (Hot North; APP1131932).

Competing interests None declared.

Patient consent for publication Obtained.

Ethics approval This study protocol is approved by the local medical ethical review committees at the University of Western Australia (Reference RA/4/20/4123), the Child and Adolescent Health Service (Approval number RGS0000000584) at Perth Children's Hospital and by the Western Australian Aboriginal Health Ethics 
Committee (Reference number: 819). This article summarises the approved trial protocol in use at the time of submission (version 7.0, dated 17 April August 2019). Ethics approval will be sought for any future protocol amendments. The study has also been reviewed and approved by the Catholic Education Office (Ref. no. RP2017/57) and Western Australian Department of Education (Ref. no. D18/0281633) for school-based surveillance. The study has also been reviewed and supported by the Kimberley Aboriginal Health Planning Forum Research Subcommittee (Ref. no. 2016-14).

\section{Provenance and peer review Not commissioned; externally peer reviewed.}

Open access This is an open access article distributed in accordance with the Creative Commons Attribution Non Commercial (CC BY-NC 4.0) license, which permits others to distribute, remix, adapt, build upon this work non-commercially, and license their derivative works on different terms, provided the original work is properly cited, appropriate credit is given, any changes made indicated, and the use is non-commercial. See: http://creativecommons.org/licenses/by-nc/4.0/.

\section{REFERENCES}

1. Australians Together. Indigenous kinship: the heart of Indigenous society. Available: https://australianstogether.org.au/discover/ indigenous-culture/kinship/ [Accessed 10 Dec 2018].

2. Currie BJ, Carapetis JR. Skin infections and infestations in Aboriginal communities in northern Australia. Australas J Dermatol 2000;41:139-43.

3. Carapetis JR, Connors C, Yarmirr D, et al. Success of a scabies control program in an Australian aboriginal community. Pediatr Infect Dis J 1997;16:494-9.

4. Davis JS, McGloughlin S, Tong SYC, et al. A novel clinical grading scale to guide the management of crusted scabies. PLoS Negl Trop Dis 2013;7:e2387.

5. Kearns TM, Speare R, Cheng AC, et al. Impact of an ivermectin mass drug administration on scabies prevalence in a remote Australian Aboriginal community. PLoS Negl Trop Dis 2015;9:e0004151.

6. Bowen AC, Mahé A, Hay RJ, et al. The global epidemiology of impetigo: a systematic review of the population prevalence of impetigo and pyoderma. PLoS One 2015;10:e0136789.

7. May PJ, Bowen AC, Carapetis JR. The inequitable burden of group A streptococcal diseases in Indigenous Australians. Med J Aust 2016;205:201-3.

8. Romani L, Steer AC, Whitfeld MJ, et al. Prevalence of scabies and impetigo worldwide: a systematic review. Lancet Infect Dis 2015;15:960-7.

9. Gear RJ, Carter JC, Carapetis JR, et al. Changes in the clinical and epidemiological features of group A streptococcal bacteraemia in Australia's Northern Territory. Trop Med Int Health 2015;20:40-7.

10. Carapetis JR, Walker AM, Hibble M, et al. Clinical and epidemiological features of group A streptococcal bacteraemia in a region with hyperendemic superficial streptococcal infection. Epidemiol Infect 1999;122:59-65.

11. Skull SA, Krause V, Coombs G, et al. Investigation of a cluster of Staphylococcus aureus invasive infection in the top end of the Northern Territory. Aust N Z J Med 1999;29:66-72.

12. Engelman D, Hofer A, Davis JS, et al. Invasive Staphylococcus aureus infections in children in tropical northern Australia. J Pediatric Infect Dis Soc 2014;3:304-11.

13. Martin AC, Anderson D, Lucey J, et al. Predictors of outcome in pediatric osteomyelitis: five years experience in a single tertiary center. Pediatr Infect Dis J 2016;35:387-91.

14. Brischetto A, Leung G, Marshall CS, et al. A retrospective CaseSeries of children with bone and joint infection from Northern Australia. Medicine 2016;95:e2885.

15. Morgan DS, Fisher D, Merianos A, et al. An 18 year clinical review of septic arthritis from tropical Australia. Epidemiol Infect 1996;117:423-8.

16. Gillespie WJ. The epidemiology of acute haematogenous osteomyelitis of childhood. Int J Epidemiol 1985;14:600-6.

17. Marshall CS, Cheng AC, Markey PG, et al. Acute post-streptococcal glomerulonephritis in the Northern Territory of Australia: a review of 16 years data and comparison with the literature. Am J Trop Med Hyg 2011;85:703-10.

18. Hoy WE, White AV, Dowling A, et al. Post-streptococcal glomerulonephritis is a strong risk factor for chronic kidney disease in later life. Kidney Int 2012;81:1026-32.

19. McDonald M, Currie BJ, Carapetis JR. Acute rheumatic fever: a chink in the chain that links the heart to the throat? Lancet Infect Dis 2004;4:240-5.
20. Carapetis JR, Steer AC, Mulholland EK, et al. The global burden of group A streptococcal diseases. Lancet Infect Dis 2005;5:685-94.

21. Hendrickx D, Bowen AC, Marsh JA, et al. Ascertaining infectious disease burden through primary care clinic attendance among young Aboriginal children living in four remote communities in Western Australia. PLoS One 2018;13:e0203684.

22. Abdalla T, Hendrickx D, Fathima $P$, et al. Hospital admissions for skin infections among Western Australian children and adolescents from 1996 to 2012. PLoS One 2017;12:e0188803.

23. Yeoh DK, Anderson A, Cleland G, et al. Are scabies and impetigo "normalised"? A cross-sectional comparative study of hospitalised children in northern Australia assessing clinical recognition and treatment of skin infections. PLoS Negl Trop Dis 2017;11:e0005726.

24. Copas AJ, Lewis JJ, Thompson JA, et al. Designing a stepped wedge trial: three main designs, carry-over effects and randomisation approaches. Trials 2015;16.

25. Hemming K, Haines TP, Chilton PJ, et al. The stepped wedge cluster randomised trial: rationale, design, analysis, and reporting. BMJ 2015;350:h391

26. Hussey MA, Hughes JP. Design and analysis of stepped wedge cluster randomized trials. Contemp Clin Trials 2007;28:182-91.

27. Hay R, Andersson N, Estrada R. Mexico: community dermatology in Guerrero. Lancet 1991;337:906-7.

28. Bowen AC, Tong SYC, Andrews RM, et al. Short-course oral cotrimoxazole versus intramuscular benzathine benzylpenicillin for impetigo in a highly endemic region: an open-label, randomised, controlled, non-inferiority trial. Lancet 2014;384:2132-40.

29. Romani L, Whitfeld MJ, Koroivueta J, et al. Mass drug administration for scabies control in a population with endemic disease. $N$ Engl $J$ Med 2015;373:2305-13.

30. One Disease. Managing crusted scabies in remote Aboriginal communities: chronic disease case management of crusted scabies to break the cycle of recurrences and transmission, 2014.

31. Coffey PM, Ralph AP, Krause VL. The role of social determinants of health in the risk and prevention of group A streptococcal infection, acute rheumatic fever and rheumatic heart disease: a systematic review. PLoS Negl Trop Dis 2018;12:e0006577.

32. RHD Action. National healthy skin guideline: for the prevention, treatment and public health control of impetigo, scabies, crusted scabies and tinea for Indigenous populations and communities in Australia. 1st edn, 2018.

33. Kimberley skin infection protocol. 2014

34. National healthy skin guideline website. Available: https:// infectiousdiseases.telethonkids.org.au/our-research/skin-guidelines/ [Accessed 28 Sep 2018].

35. Pharmaceutical Benefits Advisory Committee. Public summary document: ivermectin, $3 \mathrm{mg}$, tablet, Stromectol, 2014.

36. Public Health Act. WA, 2016.

37. Dudgeon P, Scrine C, Cox A, et al. Facilitating Empowerment and Self-Determination through participatory action research: findings from the National Empowerment project. Int J Qual 2017;16.

38. Markiewicz A, Patrick I. Developing monitoring and evaluation frameworks. USA: SAGE Publications, 2016.

39. McCawley P. The logic model for program planning and evaluation. $J$ Ext 2002;47.

40. Read C, Mitchell AG, de Dassel JL, et al. Qualitative evaluation of a complex intervention to improve rheumatic heart disease secondary prophylaxis. J Am Heart Assoc 2018;7.

41. Baio G, Copas A, Ambler G, et al. Sample size calculation for a stepped wedge trial. Trials 2015;16:354.

42. Woertman W, de Hoop E, Moerbeek M, et al. Stepped wedge designs could reduce the required sample size in cluster randomized trials. J Clin Epidemiol 2013;66:752-8.

43. Barker D, McElduff P, D'Este C, et al. Stepped wedge cluster randomised trials: a review of the statistical methodology used and available. BMC Med Res Methodol 2016;16:69.

44. Kenward MG, Roger JH. Small sample inference for fixed effects from restricted maximum likelihood. Biometrics 1997;53:983-97.

45. Gelman A. Prior distributions for variance parameters in hierarchical models (Comment on article by Browne and Draper). Bayesian Anal 2006;1:515-34

46. Medrio. Available: https://medrio.com [Accessed 24 Nov 2018].

47. Harris PA, Taylor R, Thielke R, et al. Research electronic data capture (REDCap) - a metadata-driven methodology and workflow process for providing translational research informatics support. J Biomed Inform 2009;42:377-81.

48. Bowen AC, Lilliebridge RA, Tong SYC, et al. Is Streptococcus pyogenes resistant or susceptible to trimethoprim-sulfamethoxazole? J Clin Microbiol 2012;50:4067-72.

49. Bowen AC, Tong SYC, Chatfield MD, et al. The microbiology of impetigo in Indigenous children: associations between 
Streptococcus pyogenes, Staphylococcus aureus, scabies, and nasal carriage. BMC Infect Dis 2014;14:727.

50. Biddle N, Prout S. The geography and demography of Indigenous temporary mobility: an analysis of the 2006 census snapshot. $J$ Popul Res 2009;26:305-26.

51. Andrews RM, Kearns T, Connors C, et al. A regional initiative to reduce skin infections amongst aboriginal children living in remote communities of the Northern Territory, Australia. PLoS Negl Trop Dis 2009;3:e554.

52. Wong L-C, Amega B, Barker R, et al. Factors supporting sustainability of a community-based scabies control program. Australas J Dermatol 2002;43:274-7.

53. Malyon R, Zhao Y, Guthridge S, eds. Health workforce modelling, Northern Territory, technical report for the medical workforce model. Northern Territory: Department of Health and Families, 2010.

54. Boulton J, ed. Aboriginal children, history, and health: beyond social determinants. London: Routledge, 2016.

55. Atkinson J, Nelson J, Brooks R, et al. Addressing individual and community transgenerational trauma, in Working together: Aboriginal and Torres Strait Islander mental health and wellbeing principles and practice. In: Dudgeon P, Milroy H, Walker R, et al, eds. Working together: Aboriginal and Torres Strait Islander mental health and wellbeing principles and practice. Canberra: Department of The Prime Minister and Cabinet, 2014: 289-306.

56. Fogarty W, Lovell M, Langenberg J, et al. Deficit discourse and strengths-based approaches: changing the narrative of Aboriginal and Torres Strait Islander health and wellbeing. Canberra: National Centre for Indigenous Studies, Australian National University and Lowitja Institute, 2018.

57. Wong LC, Amega B, Connors C, et al. Outcome of an interventional program for scabies in an Indigenous community. Med J Aust 2001;175:367-70.

58. Taplin D, Porcelain SL, Meinking TL, et al. Community control of scabies: a model based on use of permethrin cream. Lancet 1991;337:1016-8.

59. Ethical conduct in research with Aboriginal and Torres Strait Islander Peoples and communities. Guidelines for researchers and stakeholders. Canberra: National Health and Medical Research Council, 2018. 